\title{
Non-coding RNAs Function as Immune Regulators in Teleost Fish
}

\author{
Man Wang ${ }^{1}$, Shuai Jiang ${ }^{2}$, Wei Wu ${ }^{1}$, Fei Yu ${ }^{1}$, Wenguang Chang ${ }^{1}$, Peifeng $\mathrm{Li}^{1 *}$ and \\ Kun Wang ${ }^{1 *}$ \\ ${ }^{1}$ Institute for Translational Medicine, Medical College of Qingdao University, Qingdao, China, ${ }^{2}$ Key Laboratory of \\ Experimental Marine Biology, Institute of Oceanology, Chinese Academy of Sciences, Qingdao, China
}

OPEN ACCESS

Edited by:

Brian Dixon,

University of Waterloo, Canada

Reviewed by:

Paul M. Craig,

University of Waterloo, Canada

Shyh-Jye Lee,

College of Life Science, National

Taiwan University, Taiwan

*Correspondence:

Peifeng $L$

peifli@qdu.edu.cn

Kun Wang

wangk696@163.com

Specialty section:

This article was submitted to

Comparative Immunology,

a section of the journal

Frontiers in Immunology

Received: 28 August 2018 Accepted: 13 November 2018

Published: 28 November 2018

Citation:

Wang $M$, Jiang S, Wu W, Yu F Chang W, Li P and Wang K (2018)

Non-coding RNAs Function as

Immune Regulators in Teleost Fish.

Front. Immunol. 9:2801.

doi: 10.3389/fimmu.2018.02801
Non-coding RNAs (ncRNAs) are functional RNA molecules that are transcribed from DNA but not translated into proteins. ncRNAs function as key regulators of gene expression and chromatin modification. Recently, the functional role of ncRNAs in teleost fish has been extensively studied. Teleost fish are a highly diverse group among the vertebrate lineage. Fish are also important in terms of aquatic ecosystem, food production and human life, being the source of animal proteins worldwide and models of biomedical research. However, teleost fish always suffer from the invasion of infectious pathogens including viruses and bacteria, which has resulted in a tremendous economic loss to the fishing industry worldwide. Emerging evidence suggests that ncRNAs, especially miRNAs and IncRNAs, may serve as important regulators in cytokine and chemokine signaling, antigen presentation, complement and coagulation cascades, and $\mathrm{T}$ cell response in teleost fish. In this review, we summarize current knowledge and understanding of the roles of both miRNAs and IncRNAs in immune regulation in teleost fish. Molecular mechanism insights into the function of ncRNAs in fish immune response may contribute to the development of potential biomarkers and therapeutic targets for the prevention and treatment of fish diseases.

Keywords: non-coding RNA, fish, infectious pathogen, immune response, immune regulator

\section{INTRODUCTION}

Teleosts represent the largest and most diverse group of vertebrates, with an estimated number of species exceeding 25,000 (1). Teleosts are crucial in broad terms of ecology and food production. Teleosts also serve as biological models for developmental biology and genomic studies due to their high fecundity, rapid development, and ease of genetic manipulation $(2,3)$. However, teleost fish always suffer from the invasion of infectious pathogens including viruses and bacteria, which has caused a tremendous economic loss to the fishing industry worldwide. Therefore, it is urgent to identify effective biomarkers and therapeutic targets for the prevention and treatment of infectious fish diseases.

Non-coding RNAs (ncRNAs) are functional RNA molecules that are generally not translated into proteins (4). A growing body of evidence indicates that ncRNAs play a crucial role in diverse biological processes, such as development, differentiation and epigenetic regulation (5-7). In recent years, the research of teleost ncRNAs has been rapidly developed. Reportedly, teleost miRNAs are involved in multiple biological processes, including development, organogenesis, regeneration, tissue differentiation, growth and responses to environmental stimuli (8-11). LncRNAs have been found to be correlated with development and differentiation in teleost fish $(12,13)$. More 
importantly, the functional role of miRNAs and lncRNAs in fish immune responses has also been disclosed in several species (13-15). miRNAs and lncRNAs may serve as critical regulators in cytokine and chemokine signaling, antigen presentation, complement and coagulation cascades, and $\mathrm{T}$ cell response in teleost fish. In this review, we focus on the functional roles of miRNAs and lncRNAs in the regulation of fish immune responses. The advancement of our understanding about the immunoregulatory function of ncRNAs in teleost fish would facilitate the development of potential diagnostic markers and therapeutic targets for pathogenic diseases in economically important teleost fish species.

\section{THE CLASSIFICATION AND FUNCTION OF NCRNAS}

ncRNAs are mainly grouped into short/small ncRNAs and long ncRNAs (lncRNAs) based on their length (16). ncRNAs shorter than 200 nucleotides are usually defined as short/small ncRNAs, which include microRNAs (miRNAs), small nuclear RNAs (snRNAs), small nucleolar RNAs (snoRNAs), small interfering RNAs (siRNAs), transfer RNAs (tRNAs), and piwi-interacting RNAs (piRNAs) $(17,18)$. The most studied class of short ncRNAs is the miRNAs. miRNAs are single-stranded RNA molecules composed of approximately 18-24 nucleotides and can control gene expression by specifically targeting mRNAs (19). LncRNAs, which are usually longer than 200 nucleotides, are classified as long intronic ncRNAs, long intergenic ncRNAs, and natural antisense transcripts (20-23). Recently, a new group of ncRNAs, called circular RNAs (circRNAs), has been uncovered in various species (24-27). The length range and biological function of each type of ncRNAs are shown in Table 1 . Their best characterized function is gene regulation. miRNAs can regulate gene expression by causing mRNA degradation or translational suppression (46). Like miRNAs, siRNAs, and piRNAs are able to mediate gene silencing by base pairing to the specific region of target mRNAs (47). LncRNAs are able to antagonize miRNA-mediated gene silencing by acting as competing endogenous RNAs (ceRNAs) (48).

\section{THE FUNCTIONAL ROLE OF MIRNAS IN FISH IMMUNE REGULATION}

To date, a variety of miRNAs have been identified to be dysregulated in teleost fish during pathogen infection. Target genes of the aberrantly expressed miRNAs are prognosed through bioinformatics analysis. These dysregulated miRNAs might regulate the expression of genes that are involved in chemokine/cytokine signaling, TLR signaling, inflammatory response, complement and coagulation cascades, B- and T-cell mediated immune responses. Moreover, a few miRNAs have been further characterized by studying their effect on target genes (Table 2). Thus, increasing evidence confirms the important role of miRNAs in fish immune regulation. Further research is still needed to fully elucidate the regulatory function of miRNAs in fish immune response against pathogen infection.
TABLE 1 | The category and function of non-coding RNAs.

\begin{tabular}{|c|c|c|c|}
\hline $\begin{array}{l}\text { Non-coding } \\
\text { RNA }\end{array}$ & Size & Functions & References \\
\hline miRNAs & $18-24 \mathrm{nt}$ & $\begin{array}{l}\text { Post-transcriptional } \\
\text { regulation }\end{array}$ & $(28,29)$ \\
\hline snRNAs & $\begin{array}{c}100-300 \\
\mathrm{nt}\end{array}$ & Pre-mRNA processing & $(30,31)$ \\
\hline snoRNAs & $60-300 \mathrm{nt}$ & Nucleotide modification & $(32-34)$ \\
\hline siRNAs & $20-30 \mathrm{nt}$ & RNA interference & $(35,36)$ \\
\hline tRNAs & $74-95 \mathrm{nt}$ & $\begin{array}{l}\text { Transfer of amino acids } \\
\text { during protein synthesis }\end{array}$ & $(37,38)$ \\
\hline piRNAs & $24-32 \mathrm{nt}$ & $\begin{array}{l}\text { Translational suppression, } \\
\text { epigenetic regulation, } \\
\text { transposon repression }\end{array}$ & $(39-41)$ \\
\hline IncRNAs & $>200 \mathrm{nt}$ & $\begin{array}{l}\text { Gene expression regulation, } \\
\text { epigenetic and chromatin } \\
\text { structure modifications }\end{array}$ & $(42-44)$ \\
\hline CircRNAs & Circular & $\begin{array}{l}\text { Gene expression regulation, } \\
\text { miRNA sponge }\end{array}$ & $(24,45)$ \\
\hline
\end{tabular}

\section{Differentially Expressed miRNAs Related to Viral Challenge}

The miRNAs responsive to the viral mimic, polyriboinosinic polyribocytidylic acid (poly I:C), were identified in Atlantic cod through deep sequencing technology (14). The expression of ten miRNAs, including miR-731-3p, miR-462-3p, miR-21883p, miR-125b-3-3p, miR-150-3p, miR-128-3-5p, miR-214-1-5p, miR-451-3p, miR-30b-3p, and miR-199-1-3p, was confirmed to be altered upon poly I:C stimulation. Moreover, these differentially expressed miRNAs were predicted to target immune-related genes, such as interferon (IFN)-stimulated gene (ISG), CXC chemokine, and cytotoxic and regulatory T-cell protein precursor. Nevertheless, the number of sequencing reads mapped to miRNAs in the poly I:C stimulated samples was lower compared to the controls, which might affect the sensitivity of miRNA detection in Atlantic cod. Accordingly, several significantly deregulated miRNAs (e.g., miR-128-3-5p, miR-214$1-5 p$, and miR-731-3p) were not identified as differentially expressed by bioinformatics analysis. Remarkably, miR-462-3p, miR-731-3p, and miR-2188-3p have been reported as virusresponsive in several teleost species (72-74), suggesting that the three miRNAs were teleost-specific miRNAs upregulated by viral mimic stimulation. These miRNAs might exert similar functions in antiviral immune responses in teleost fish. The function of the identified miRNAs awaits further validation through loss- and gain-of-functional assays. Megalocytivirus, a highly infectious DNA virus, is a serious pathogen to a wide range of marine fish including Japanese flounder, catfish and rainbow trout (75-77). The expression profile of miRNAs was explored in Japanese flounder infected with megalocytivirus (78). The result indicated that 121 host miRNAs and 9 viral miRNAs were differentially expressed in virus-infected Japanese flounder. Functional categories showed that target genes of these miRNAs were predicted to be involved in immune response and apoptotic process. Specifically, apoptosis is an innate response of the host to counteract invading pathogens (79). The flounder miRNAs 
TABLE 2 | Overview of miRNAs characterized in teleost fish.

\begin{tabular}{|c|c|c|c|c|}
\hline miRNAs & Fish species & Targets & Function & References \\
\hline cse-miR-146a & Half-smooth tongue sole (Cynoglossus semilaevis) & IL-1 $\beta$ & Regulate inflammatory response & $(49)$ \\
\hline cse-miR-33 & Half-smooth tongue sole (Cynoglossus semilaevis) & caspase-8 & Regulate cell apoptosis & $(49)$ \\
\hline cse-let-7 & Half-smooth tongue sole (Cynoglossus semilaevis) & IGFBP1 & Regulate cell apoptosis & $(49)$ \\
\hline $\operatorname{miR}-148$ & Half-smooth tongue sole (Cynoglossus semilaevis) & SOCS7 & Regulate cytokine signaling & (49) \\
\hline cse-miR-143 & Half-smooth tongue sole (Cynoglossus semilaevis) & ATG2B & Regulate cell autophagy & $(49)$ \\
\hline cse-miR-152 & Half-smooth tongue sole (Cynoglossus semilaevis) & NLRC5 & Regulate antigen processing & $(49)$ \\
\hline cse-miR-23a & Half-smooth tongue sole (Cynoglossus semilaevis) & STAT1 & Regulate IFN signaling & (49) \\
\hline cse-miR-26a & Half-smooth tongue sole (Cynoglossus semilaevis) & WIPI1 & Regulate cell autophagy & $(49)$ \\
\hline cse-miR-71c-5p & Half-smooth tongue sole (Cynoglossus semilaevis) & IL-10 & Regulate inflammatory response & $(49)$ \\
\hline cse-miR-8192-3p & Half-smooth tongue sole (Cynoglossus semilaevis) & WIPI1 & Regulate cell autophagy & $(49)$ \\
\hline $\mathrm{miR}-148$ & Miiuy croaker (Miichthys miiuy) & MyD88 & Regulate NF-кB signaling pathway & $(50)$ \\
\hline miR-214 & Miiuy croaker (Miichthys miiuy) & MyD88 & Regulate NF-кB signaling pathway & $(51)$ \\
\hline $\operatorname{miR}-3570$ & Miiuy croaker (Miichthys miiuy) & MyD88; MAVS & Regulate NF-кB and IRF3 signaling pathways & $(52,53)$ \\
\hline miR-19a & Miiuy croaker (Miichthys miiuy) & MyD88 & Regulate NF-кB signaling pathway & $(54)$ \\
\hline miR-192 & Miiuy croaker (Miichthys miiuy) & $|L-1 R|$ & Regulate inflammatory response & $(55)$ \\
\hline miR-216a & Miiuy croaker (Miichthys miiuy) & p65 & Regulate NF-кB signaling pathway & $(15)$ \\
\hline miR-203 & Miiuy croaker (Miichthys miiuy) & IRAK4 & Regulate NF-кB signaling pathway & $(56)$ \\
\hline miR-375 & Miiuy croaker (Miichthys miiuy) & DUSP1 & Regulate NF-кB signaling pathway & $(57)$ \\
\hline $\operatorname{miR}-146 a$ & Fathead minnow (Pimephales promelas) & ND & Regulate cell apoptosis and NF-кB signaling pathway & $(58)$ \\
\hline $\operatorname{miR}-210$ & Miiuy croaker (Miichthys miiuy) & DUBA & Regulate RIG-I signaling pathway & $(59)$ \\
\hline miR-210 & Miiuy croaker (Miichthys miiuy) & STING & Regulate type I IFN signaling pathway & $(60)$ \\
\hline pol-miR-731 & Japanese flounder (Paralichthys olivaceus) & IRF7, p53 & Regulate type I IFN response and cell apoptosis & $(61)$ \\
\hline $\operatorname{miR}-152$ & Antarctic ice-fish (Chionodraco hamatus) & GATA1 & Regulate hematopoiesis & $(62)$ \\
\hline miR-146a & Orange spotted grouper (Epinephelus coioides) & TRAF6 & Regulate inflammatory response & (63) \\
\hline miR-126-03 & Atlantic salmon (Salmo salar) & IFNg & Regulate IFN signaling pathway & $(64)$ \\
\hline miR-214 & Snakehead fish (Channa striatus) & Viral N/P genes & Regulate SHW propagation & $(65)$ \\
\hline $\operatorname{miR}-8159$ & Miiuy croaker (Miichthys miiuy) & TLR13 & Mediate TLR signaling pathway & $(66)$ \\
\hline $\operatorname{miR}-8159-5 p$ & Miiuy croaker (Miichthys miiuy) & TLR1 & Mediate TLR signaling pathway & $(67)$ \\
\hline $\operatorname{miR}-217-5 p$ & Miiuy croaker (Miichthys miiuy) & TLR1 & Mediate TLR signaling pathway & $(67)$ \\
\hline miR-200a-3p & Miiuy croaker (Miichthys miiuy) & TLR1 & Mediate TLR signaling pathway & (68) \\
\hline miR-122 & Miiuy croaker (Miichthys miiuy) & TLR14 & Mediate TLR signaling pathway & (69) \\
\hline $\operatorname{miR}-21$ & Miiuy croaker (Miichthys miiuy) & TLR28 & Mediate TLR signaling pathway & $(70)$ \\
\hline cid-miRn-115 & Grass carp (Ctenopharyngodon idella) & TLR5 & Mediate TLR signaling pathway & $(71)$ \\
\hline $\operatorname{miR}-142 a-3 p$ & Grass carp (Ctenopharyngodon idella) & TLR5 & Mediate TLR signaling pathway & $(71)$ \\
\hline
\end{tabular}

ND, not determined.

could target both pro- and anti-apoptotic genes, suggesting that some of the flounder miRNAs functioned in enhancing host defense responses, while others might operate to promote viral infection. It could be speculated that the differentially expressed host miRNAs might act on the host itself or virus. The host miRNAs might actively exert their functions in a natural manner that favored viral clearance. Oppositely, the host miRNAs could be subverted by the virus to promote viral infection. The effect of viral hemorrhagic septicemia virus (VHSV) infection on the miRNA expression profile in olive flounder was also studied (72). A total of 372 miRNAs were found, and 63 of these miRNAs were differentially expressed during VHSV infection. The predicted target genes of differentially expressed miRNAs were implicated in immune-related pathways such as cytokine-cytokine receptor interaction, nuclear factor $\kappa \mathrm{B}(\mathrm{NF}-\kappa \mathrm{B})$ signaling pathway and tumor necrosis factor (TNF) signaling pathway. Notably, the most strikingly increased miRNAs during VHSV infection was
miR-155. The putative target genes for miR-155 included the complement component C9 and the heat shock protein 90 (HSP90). HSP90 works as a molecular chaperone that promotes correct folding of target proteins (80). More importantly, HSP90 is also essential for viral replication $(81,82)$. The significant upregulation of HSP90-targeting miRNAs could be favorable or detrimental to olive flounder. Therefore, further studies should be conducted to disclose the role of the miRNA/HSP90 regulatory pathway in VHSV pathogenesis.

A total of 116 miRNAs were previously identified in Epinephelus coioides by the high-throughput sequencing approach (83). Among these miRNA, 107 miRNAs were identical in sequence to that of zebrafish. Moreover, 65 miRNAs had homologs in human, mouse, rat and pufferfish. The identified miRNAs in grouper were phylogenetically conserved. In the process of Singapore grouper iridovirus (SGIV) infection, 40 differentially expressed miRNAs were identified in $E$. 
coioides. During viral infection, host miRNAs underwent altered expression profiles, which led to feed-back or feed-forward effects on viral infection (84). The differentially expressed miRNAs were implicated in immune-related pathways including antigen processing and delivery, natural killer cell mediated cytotoxicity, and chemokine signaling pathway. These results might provide useful clues to uncover the mechanisms of host-virus interactions during SGIV infection. A total of 205 differentially expressed miRNAs were identified in snakehead fish vesiculovirus (SHVV)-infected snakehead fish cell line (85). Specifically, three differentially expressed miRNAs, including miR-130-5p, miR-214, and miR-216b, could inhibit the expression of viral genes. These miRNAs displayed significant antiviral properties. The three miRNAs might also target host genes that were involved in antiviral signaling pathways. It seemed that teleost miRNAs utilized both direct and indirect ways to inhibit SHVV multiplication. However, the detailed mechanisms underlying the inhibition of SHVV replication by teleost miRNAs remain to be further characterized. Another study revealed the miRNA response in rainbow trout following VHSV challenge (86). Two clustered miRNAs, miR-462, and miR-731, were strongly induced in rainbow trout following VHSV infection, indicating their engagement in the fish-virus interaction. In rainbow trout, the expression of miR-462 and miR-731 was positively correlated with the levels of IFNs and IFN-induced genes (87). Further study indicated that IFN stimulation could upregulate the expression of the two miRNAs, suggesting that the induction of miR-462 and miR-731 was elicited by IFNs. Knockdown of these miRNAs in rainbow trout treated with the IFN inducer poly I:C crippled the ability of poly I:C to protect fish against lethal VHSV infection. These findings implied that miR-462 and miR-731 might be involved in IFN-mediated antiviral defense in teleost fish. The true targets of these two miRNAs are currently undetermined and their regulatory effect on viral gene expression deserves further exploration. The miRNA response was identified in Atlantic salmon infected with salmonid alphavirus (SAV) (74). A total of 20 miRNAs showed altered expression during SAV infection. The differentially expressed miRNAs were predicted to target a variety of immune genes, such as IFN regulatory factors, $\mathrm{C}-\mathrm{C}$ motif chemokines, cytokines, and NF- $\kappa \mathrm{B}$ inhibitors. This study demonstrated that miRNAs functioned as inhibitors of harmful inflammation as well as promoters of early immune responses. The expression of these dysregulated miRNAs might differ depending on what kind of virus subtype that caused host immune responses. Additionally, the correctness of target gene prediction remains to be defined.

The expression pattern of miRNAs was characterized in epithelioma papulosum cyprini (EPC) cells following spring viremia of carp virus (SVCV) infection (88). A total of 14 miRNAs were found to be differentially expressed. Among their target genes, 51 genes were associated with IFN, interleukin, TNF and Toll-like receptor (TLR), all of which played important roles in host antiviral responses. Some differentially expressed miRNAs were predicted to target the coding region (CDS) of the SVCV genes, suggesting that these miRNAs might affect SVCV infection. Additionally, high-throughput sequencing combined with bioinformatics analysis was employed to characterize the function of immune-related miRNAs in miiuy croaker with poly I:C stimulation (89). Several upregulated miRNAs (e.g., mmi-miR-155, mmi-miR-181a-3p, mmi-miR-19a-3p, and mmimiR-132-3p) identified in miluy croaker had the potential to target inhibiting factors in the RIG-I like receptor (RLR) signaling pathway, thus, these miRNAs indirectly activated the signaling pathway. Moreover, miR-184, miR-200b-3p, and miR$30 \mathrm{a}-5 \mathrm{p}$ were predicted to negatively regulate the expression of caspase-8 (CASP8), c-Jun N-terminal kinase (JNK), IFN regulatory factor 3 (IRF3), and p38 mitogen-activated protein kinase (p38 MAPK). CASP8 participates in the regulation of apoptosis, while JNK, IRF3, and p38 MAPK can modulate cytokine production (90-92). miR-184, miR-200b-3p, and miR$30 a-5 p$ were downregulated in miiuy croaker, thus weakening their inhibitory effect on the target genes. Based on these results, miRNAs could indirectly activate fish immune responses through suppressing the inhibitory factor in signaling pathways. Moreover, attenuating miRNA-mediated silencing of immunerelated genes resulted in the direct enhancement of fish immune responses. This study provided the opportunity to deeply understand the functional roles of miRNAs in fish immune regulation.

In these studies, a number of differentially expressed miRNAs have been identified in teleost fish. It is likely that most of differentially expressed miRNAs are not directly associated with fish immune regulation, but respond to external stimuli due to viral challenge. Some miRNAs may be even false positives. Nevertheless, if one miRNA is identified as differentially expressed in diverse teleost species infected with different viruses, this miRNA may serve as a vital modulator in fish immune system. For instance, miRNA-462/731 cluster and miR-2188 were conserved differentially expressed miRNAs in Japanese flounder and Atlantic cod following viral challenge, suggesting that these miRNAs played an important role in fish antiviral immunity. Notably, the miRNA-462/731 cluster is teleost-specific and locates downstream of IFN-inducible and immune-related promoter elements (86), indicating its crucial role in immune responses against viral infection in teleost fish. However, most of these studies focused on identifying miRNAs that were differentially expressed between the controls and challenged groups. The differentially expressed miRNAs were candidate miRNAs that might regulate immune-relevant genes. Experimental studies are demanded to validate the true targets of the deregulated teleost miRNAs during viral infection. Further investigation of teleost miRNAs as well as their target genes will deepen our understanding of the function of miRNA/mRNA regulatory axes in fish antiviral immunity.

\section{Fish Virus-Encoded miRNAs With Immunomodulatory Functions}

Viruses have evolved their own miRNAs to regulate specific host pathways as a means for their survival and pathogenesis. The expression pattern of viral miRNAs was investigated in megalocytivirus-infected Japanese flounder (78). The result showed that nine viral miRNAs were upregulated and predicted 
to target immune-relevant genes including Toll like receptor 14 (TLR14), IRF3, and TNF receptor-1 (TNFR-1). None of the megalocytivirus miRNAs shared homology with known viral miRNAs. Viral miRNAs undergo fast evolution and mutation, which may render the virus capable of surviving in specific host environments. Yan et al. (93) found 16 novel viral miRNAs in SGIV-infected grouper cells through the high-throughput sequencing technology. These viral miRNAs were predicted to be engaged in viral proliferation and persistence, antiviral immune response and cell cycle regulation. This study might lay the foundation for further researches on SGIV pathogenesis.

Deep sequencing of the small RNAome in Cyprinus herpesvirus 3 (CyHV-3)-infected carps (Cyprinus carpio) revealed that $\mathrm{CyHV}-3$ was able to encode two pre-miRNAs (94). Mature miRNAs from the two pre-miRNAs might target viral dUTPase. Since viral dUTPase can function as a signaling molecule to regulate antiviral immune responses (95), CyHV-3-encoded miRNAs might be involved in host immune regulation. CyHV-3 miRNAs might also target host mRNAs. The host target genes of CyHV-3 miRNAs could be identified once the $C$. carpio genome has been fully annotated. Among the SGIV miRNAs, SGIV-miR-13 exhibited enhanced expression during the early stage of SGIV infection (96). Overexpression of SGIV-miR-13 could significantly inhibit viral replication by targeting its major capsid protein (SGIVMCP). Therefore, SGIV-miR-13 could effectively block host antiviral responses by restricting early viral replication. These results demonstrated that viruses have evolved their miRNA repertoire to escape host immune surveillance. At the late stage of viral infection, the expression of SGIV-miR-13 was decreased, thus relieving its inhibition of SGIV-MCP. Consequently, the downregulation of SGIV-miR-13 benefited viral replication. There was a tightly regulated kinetics of viral miRNA-mediated gene silencing during SGIV infection. Systematic explorations of SGIV-encoded miRNAs would provide novel insights into the molecular mechanism of SGIV pathogenesis. Host cells usually undergo apoptosis in response to viral infection, leading to reduced production and release of progeny virus (97). Viruses have evolved to manipulate host cell apoptosis. For instance, SGIV-encoded miR-homoHSV targeted viral pro-apoptotic lipopolysaccharide-induced TNF- $\alpha$ (LITAF)like factor and suppressed LITAF-induced apoptosis (98). Therefore, miR-homoHSV inhibited SGIV-induced cell death and facilitated viral replication by targeting LITAF. These data demonstrated that viral miRNAs and protein-coding genes might work synergistically in a complicated manner to favor viral pathogenesis.

\section{Altered Expression of miRNAs in Response to Bacterial Challenge}

Yuhong et al. (99) previously identified immune-related miRNAs in Megalobrama amblycephala using the highthroughput sequencing technology. As a result, 324 miRNAs were discovered, with 63 showed increased expression levels in lipopolysaccharide (LPS)-stimulated M. amblycephala. Functional annotation indicated that most of differentially expressed miRNAs were associated with immune system development and immune response, such as hematopoietic or lymphoid organ development, B or T cell-mediated immunity, TLR signaling pathway, complement and chemokine signaling pathway, and antigen processing and presentation. These results suggested that the differentially expressed miRNAs in $M$. amblycephala acted as important players in innate and adaptive immune responses against bacterial infection. Identification and functional characterization of immune-related miRNAs in $M$. amblycephala would be helpful for comprehensively disclosing the role of miRNAs in fish immune system. The miR-146 family, miR-146a, and miR-146b, was usually induced by Salmonella typhimurium or Mycobacterium marinum infection in zebrafish (100). Moreover, it was possible that the miR-146 family was involved in lipid-mediated inflammatory responses in zebrafish. Another study was conducted to investigate the miRNA expression profile in common carp infected with Flavobacterium columnare (FC) (101). Totally, 30 miRNAs were differentially expressed between the control and FC-infected common carp. These differentially expressed miRNAs were mainly enriched in pathways tightly associated with the bacterial infection, such as focal adhesion, erythroblastic leukemia viral oncogene homolog (ErbB) signaling pathway, adherent junction and regulation of actin cytoskeleton. These pathways were associated with cytoskeleton and its related signaling pathways, suggesting that FC infection exerted significant effects on cell proliferation, migration, adhesion, and differentiation in common carp. The exact mechanisms are unknown and remain to be elucidated. In addition, the differentially expressed miRNAs were also grouped into immune-related pathways including natural killer cell mediated cytotoxicity, TLR signaling pathway, transforming growth factor- $\beta$ (TGF- $\beta$ ) signaling pathway, and mTOR signaling pathway. The immune mechanisms of these miRNAs involved in FC infection in common carp need further investigation.

The expression profile of the miRNAs response to Vibrio anguillarum infection was analyzed in Cynoglossus semilaevis (102). Compared to the controls, 175 and 215 miRNAs were differentially expressed in $C$. semilaevis with nonobvious and obvious vibriosis symptoms, respectively. Functional characterization indicated that the differentially expressed miRNAs might participate in immune system development and immune responses, such as hematopoietic or lymphoid organ development, $\mathrm{T}$ and $\mathrm{B}$ cell immune responses, TLR signaling pathway and chemokine-mediated signaling pathway. These miRNAs might play a central role in the modulation of immunerelated genes in C. semilaevis during $V$. anguillarum infection. The expression pattern of miRNAs in Nile tilapia infected with Streptococcus agalactiae was also revealed (103). A total of 3,009 tilapia miRNAs were identified, and 218 miRNAs showed significantly altered expression in S. agalactiae-infected Nile tilapia. Target genes of tilapia miRNAs were prognosed to be implicated in $\mathrm{T}$ cell immune response, cytokine-mediated signaling pathway, antigen presentation, and the complement cascade. Some miRNAs (e.g., let-7, miR-21, miR-125, miR146 , and miR-155) were commonly deregulated during bacterial infection and functioned in reducing excessive inflammatory responses. let-7 and miR-125 exhibited high conservation across 
evolution in both sequence and function (104). Tilapia let7 and miR-125 might possess the same function with other species, particularly in response to bacterial infection. Moreover, different tilapia miRNAs shared some target genes. In-depth investigation of synergistic regulations among diverse miRNAs will be crucial to uncover the mechanism of complex regulatory networks in tilapia during bacterial infection. Transcriptome sequencing analysis showed that 12 miRNAs were differentially expressed between $V$. anguillarum-infected miiuy croaker and the control group (105). Target genes of the 12 significantly deregulated miRNAs might engage in TLR signaling pathway, RLR signaling pathway, cytokine-mediated signaling pathway, and $\mathrm{B}$ or $\mathrm{T}$ cell immune responses. The relationship between miiuy croaker miRNAs and their target genes in host-pathogen interactions warrants further investigation. In addition, miR-142$5 \mathrm{p}, \mathrm{miR}-223$, and miR-181a were differentially expressed in $C$. semilaevis in response to $V$. anguillarum infection (106). Target genes of these miRNAs were related to B-cells, T-cells, NF- $\kappa \mathrm{B}$, TNF, cytokines and interleukin. Therefore, these miRNAs might serve as important players in fish immune responses against bacterial infection by regulating immune-related genes. Further studies on the function of miR-142-5p, miR-223, and miR-181a would enrich our knowledge about pathogen-induced defense responses in C. semilaevis.

The expression profile of miRNAs in Edwardsiella tarda infected-tongue sole was previously reported (49). The result indicated that 311 tongue sole miRNAs showed significant differences in expression after E. tarda infection. More importantly, target genes of 10 miRNAs were confirmed. For instance, cse-miR-146a could directly target interleukin-1 $\beta$ (IL$1 \beta$ ), while miR-148 regulated the expression of suppressor of cytokine signaling 7 (SOCS7). Upregulated cse-miR-143 and cse-miR-152 targeted the autophagy-related gene $A T B 2 B$ and the NOD-like receptor (NLR) gene NLRC5, contributing to E. tarda invasion into fish tissues. NLR and autophagy are implicated in recognition/clearance of invading pathogens (107, 108). Upregulation of cse-miR-143 and cse-miRNA-152 might be a virulence strategy of $E$. tarda to escape host immune defense by targeting the related pathways. Overall, miRNAs were not only vital immune regulators in tongue sole but might also act as targets for E. tarda manipulation of host defense system. A total of 1,894 miRNAs were discovered in Streptococcus iniaeinfected tilapia (109). Among these miRNAs, seven miRNAs (miR-92d-3p, miR-127, miR-310-3p, miR-146-3p, miR-375-5p, miR-92, and miR-694) were found to be differentially expressed. Putative targets of these seven miRNAs included complement C3, cytidine deaminase, regulator of G-protein signaling 22 (Rgs22), MAPK1, calcium-sensing receptor (CaSR) and metabotropic glutamate receptor 8 (GluR8). These results suggested that tilapia miRNAs played an important role in host defense responses against bacterial infection. Further investigations are required to gain more insights into the relationship between miRNAs and their putative target genes in tilapia.

As mentioned above, teleost miRNAs may function as important regulators in fish immune responses against bacterial infection. Some teleost miRNAs, such as miR-462, miR-731, miR-181, and miR-146, were involved in the regulation of host immune responses against both bacterial and viral infection (73), suggesting that these miRNAs played a key role in host-pathogen interactions. However, target genes of multiple teleost miRNAs were predicted by computational approach. miRNA target predictions may be contaminated with a large number of false positives. The effect of predicted miRNA/mRNA regulatory axes on fish immune responses needs further functional experiments for validation.

\section{Target Genes of Teleost miRNAs Involved in Immune Regulation}

Target genes of substantial teleost miRNAs have been confirmed and are shown to be widely involved in fish immune responses, including NF- $\kappa \mathrm{B}$ signaling pathway, TLR signaling pathway and cytokine-mediated immune response (Figure 1). NF- $\kappa$ B plays a central role in activation of host pro-inflammatory response (110). Several teleost miRNAs were found to interfere with NF$\kappa \mathrm{B}$-mediated inflammatory response. The expression of miR148 (50) and miR-214 (51) was increased in miluy croaker after challenged with Vibro harveyi or LPS, and their upregulation could restrain host inflammatory responses. Moreover, miR148 and miR-214 were shown to negatively regulate the expression of myeloid differentiation primary response 88 (MyD88). miR-148 and miR-214 suppressed NF- $\kappa \mathrm{B}$ signaling pathway by directly targeting MyD88, thus avoiding excessive inflammation. Likewise, miR-3570 suppressed V. anguillaruminduced inflammatory response in miiuy croaker by inhibiting MyD88-mediated NF-кB signaling pathway (52). Another report showed that the expression of miR-3570 was induced in miluy croaker macrophages upon rhabdovirus infection (53). miR3570 directly targeted mitochondrial antiviral signaling protein (MAVS), thus leading to the blockage of MAVS-mediated NF$\kappa \mathrm{B}$ and IRF3 signaling pathway. Further study indicated that inducible miR-3570 also suppressed virus-triggered type I IFN and antiviral gene production, hence favoring rhabdovirus pathogenesis. Recently, miR-19a was also reported to restrain NF- $\kappa \mathrm{B}$ activation by targeting MyD88 in miluy croaker (54). In teleost fish, multiple miRNAs may synergistically target NF- $\mathrm{B}$ signaling pathway, hence significantly repressing host inflammatory responses.

miR-192 exhibited differential expression patterns between $V$. anguillarum challenge and healthy miiuy croakers (55). More importantly, miR-192 was confirmed to regulate inflammatory immune responses in miiuy croaker with bacterial infection by targeting IL-1 receptor type I (IL-1RI). Another two miRNAs, miR-216a (15), and miR-203 (56), were obviously upregulated in miiuy croaker challenged with $V$. anguillarum and LPS. miR-216a caused p65 downregulation by binding to its $3^{\prime}$ untranslated region ( $3^{\prime}$ UTR). Further study indicated that upregulation of miR-216a inhibited inflammatory cytokine production and blocked NF- $\mathrm{B}$ signaling pathway. Thus, miR216a functioned as a critical inhibitor of bacterial infectioninduced inflammatory responses in miiuy croaker. IL-1 receptorassociated kinase 4 (IRAK4) was the target of miR-203. miR203 repressed the activation of NF- $\kappa \mathrm{B}$ signaling pathway by reducing the expression of IRAK4. These results suggested that 


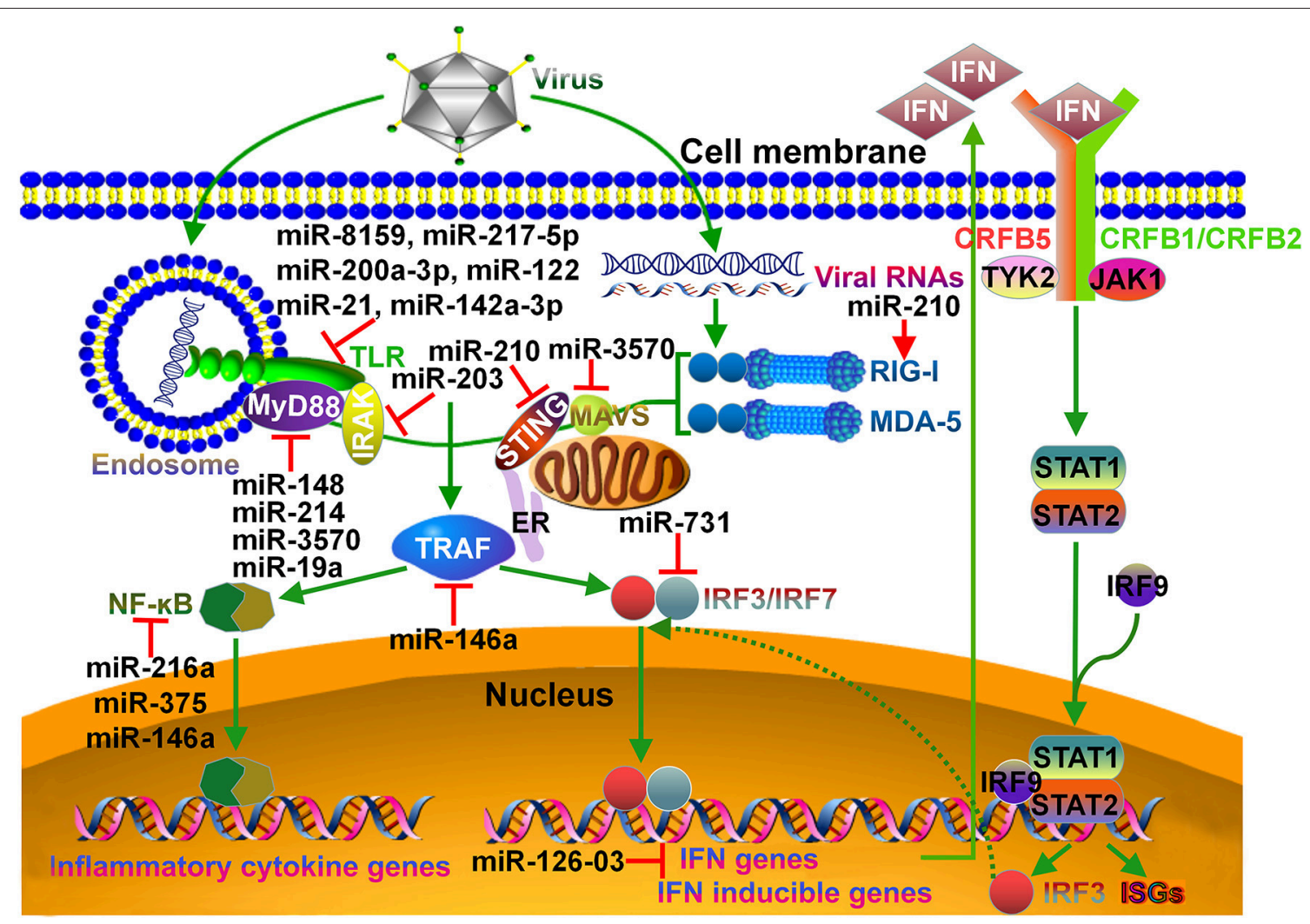

FIGURE 1 | Schematic overview of miRNA targets in fish immune system. In teleost fish, viral infection can be detected by specialized pattern recognition receptors (PRRs). Endosome localized TLRs can sense viral nucleic acids (DNA, dsRNA, ssRNA), and then deliver signal to MyD88. MyD88 forms a complex with IRAK. MyD88/IRAK can activate the downstream TRAF signaling cascade, leading to the induction of NF-kB signaling pathway and pro-inflammatory cytokine response. RIG-I-like receptors, RIG-I and MDA-5, recognize viral genomic RNAs in the cytoplasm and signal via downstream STING, MAVS and TRAF adaptors. Both STING and MAVS can induce the phosphorylation of IRF3 and IRF7. Activated IRF3 and IRF7 translocate into the nucleus and bind to DNA sequences to induce the transcription of IFNs and IFN inducible genes. The induced IFN can bind to the CRFB complex on the cell surface, resulting in the recruitment and binding of the kinases TYK2 and JAK1. These kinases catalyze the phosphorylation of STAT1 and STAT2. The IRF9/STAT1/STAT2 complex crosses the nuclear membrane and binds to IFN-stimulated response elements, thereby contributing to the transcription of IRF3 and ISGs. miRNAs that regulate particular immune pathways are indicated. TLR, Toll-like receptor; MyD88, myeloid differentiation primary response 88; IRAK, interleukin-1R-associated kinase; STING, stimulator of IFN genes; ER, endoplasmic reticulum; MAVS, mitochondrial antiviral signaling protein; RIG-I, retinoid acid inducible gene-l; MDA-5, melanoma differentiation-associated gene-5; TRAF, tumor necrosis factor receptor-associated factor; IRF, IFN regulatory factor; CRFB, cytokine receptor family B; STAT, signal transducer and activator of transcription; ISG, IFN-stimulated gene.

miR-203 in miiuy croaker was a negative modulator of innate immune response against bacterial infection. Additionally, miR375 significantly inhibited the expression of dual-specificity phosphatase 1 (DUSP1) in miiuy croaker and thus resulted in the blockade of NF- $\kappa \mathrm{B}$ signaling pathway (57). Another study revealed the regulatory function of miR-146a in fathead minnow cells infected with SGIV (58). SGIV infection boosted the expression of miR-146a. miR-146a overexpression suppressed NF- $\kappa \mathrm{B}$ activation and SGIV-induced cell apoptosis, thus promoting viral proliferation. These results indicated that miR146a served a promotive role in SGIV infection by inhibiting host antiviral immune responses.

It has been reported that the expression of miR-210 was significantly upregulated in miiuy croaker upon poly I:C stimulation (59). The upregulation of miR-210 could indirectly induce the RIG-I signaling pathway and enhanced the expression of type I IFN by targeting deubiquitinating enzyme A (DUBA), thus protecting the host from viral infection. Likewise, miR-210 was found to be upregulated in Siniperca chuatsi rhabdovirus (SCRV)-infected miiuy croaker (60). However, further study indicated that miR-210 inhibited type I IFN and inflammatory cytokine production by targeting stimulator of IFN genes (STING) and repressing NF- $\mathrm{B}$, IRF3 and IFN-stimulated response element (ISRE) signaling. The regulatory function of miR-210 in STING expression was also verified in other teleosts (Larimichthys crocea and Sciaenops ocellatus). This result demonstrated the evolutionary conservation and functional consistency of miR-210 in teleosts. Type I IFNs are early host responses to viral infection and exert important roles in antiviral process. Thus, miR-210 functioned in supporting viral infection in teleost fish. It was possible that the host miR210 could be subverted by SCRV to facilitate its infection. In summary, miR-210 could target diverse genes, and its target genes might have opposite effects on viral pathogenesis. The effect of 
miR-210 on type I IFN signaling might differ depending on the type of stimulus (poly I:C or SCRV), which further indicated the complexity of miRNA-mediated regulatory networks in teleost fish. The flounder miRNA, pol-miR-731, was upregulated upon megalocytivirus challenge and promoted viral replication (61). Further study showed that pol-miR-731 blocked type I IFN response by targeting IRF7, and it also suppressed virus infection-induced splenocyte apoptosis and cell cycle arrest by targeting p53. This study revealed the immuno-suppressive role of miRNAs in the host-virus interaction. The replenishment of hemocytes by hematopoiesis was of great significance in maintaining immune homeostasis (111). miR-152 performed a crucial role in hematopoiesis during the development of Chionodraco hamatus (62). Mechanistically, miR-152 caused decreased hematopoiesis by descending the expression of GATAbinding factor 1 (GATA1). Red spotted grouper nervous necrosis virus (RGNNV) infection constantly induced the expression of miR-146a in spleen cells of orange spotted grouper, Epinephelus coioides (63). miR-146a overexpression could promote RGNNV infection, while knockdown of miR-146a expression inhibited viral infection. Moreover, miR-146a specifically bound to the $3^{\prime}$ UTR of TNF receptor-associated factor 6 (TRAF6) and thereby attenuated the expression of downstream genes, including TNF$\alpha$, IL-8, and IL-1 $\beta$. Collectively, these results showed that miR-146a exerted an inhibitory function in TRAF6-mediated inflammatory response to viral infection in orange spotted grouper. The previous study indicated that IFNg acted as a putative target of miR-126-03 in Atlantic salmon (64). Further study verified that miR-126-03 directly regulated the expression of IFNg. miR-214 was able to inhibit SHVV replication in striped snakehead cells by directly targeting viral nucleoprotein $(\mathrm{N})$ and phosphoprotein $(\mathrm{P})$ genes (65). SHVV infection led to decreased expression of miR-214. The downregulated miR-214 in turn promoted SHVV replication by enhancing viral $\mathrm{N}$ and $\mathrm{P}$ expression and reducing IFN- $\alpha$ production. This study provided new insights into the molecular mechanisms underlying fish immune responses against viral infection.

Toll-like receptors (TLRs), a group of pattern-recognition receptors, play an important role in modulating innate immune responses against invading pathogens (112). TLR13 is significantly enhanced in miiuy croaker following infection with $V$. anguillarum (66). miR-8159 was induced in miiuy croaker challenged with $V$. anguillarum and exerted a direct negative regulatory effect on TLR13. These results demonstrated that miR-8159 inhibited fish immune responses by targeting TLR13, thus facilitating bacterial infection and pathogenesis. TLR1 performs a crucial role in sensing bacterial pathogens and anti-microbial immune responses (113). miR-8159-5p and miR$217-5 p$ were found to be upregulated in miiuy croaker upon LPS stimulation (67). The two miRNAs synergistically suppressed the expression of TLR1 response to LPS stimulation. miR-200a$3 p$ could also directly target TLR1 in miiuy croaker and thus regulated fish TLR signaling pathways (68). TLR14 has only been discovered in fish and may be involved in fish immune responses against pathogen infection (114). miR-122 displayed significantly reduced expression profiles in $V$. anguillarum challenged miiuy croaker (69). miR-122 was involved in TLR cascade by targeting TLR14 and thus controlled V. anguillarum infection in miiuy croaker. TLR28 is a new member of TLRs family and plays an important role in host immune responses (115). The previous report showed that the expression of miR21 was elevated in miiuy croaker after poly I:C stimulation (70). Upregulation of miR-21 inhibited the expression of cytokines (TNF $\alpha$ and IL-6) and antiviral genes (MX1 and ISG15). miR-21 also suppressed the excessive immune response and maintained body homeostasis in miiuy croaker by directly targeting TLR28. The activation of TLR5 could modulate the expression of genes involved in innate immune responses (71). cid-miRn-115 and miR-142a-3p were differentially expressed between susceptible and resistant grass carps infected with Aeromonas hydrophila. Upregulation of cid-miRn-115 and miR-142a-3p significantly reduced the expression of TLR5, thus suppressing its downstream effector genes including IL- 8 , TNF- $\alpha$, and IL-1 $\beta$. These results demonstrated that cid-miRn-115 and miR-142a-3p played a crucial role in governing the innate immune response.

In the process of pathogen infection, the host immune responses are triggered to resist pathogen infection. However, to establish a persistent infection, pathogens have evolved their capability to escape host immune responses. In teleost fish, miRNAs serve as crucial effectors in the modulation of hostpathogen interaction networks. Intriguingly, the implication of miRNAs not only could be a part of host defense responses against pathogen infection, but host miRNAs could also be exploited by pathogens to support their replication. A better understanding of the functional roles of host miRNAs in immune regulation during pathogen invasion would shed light on the development of novel therapies for fish diseases.

\section{The Engagement of LncRNAs in Fish Immune Response}

LncRNAs have been demonstrated to function as important regulators of gene expression (116). They are able to cis-regulate the expression of their neighboring protein-coding genes (117). LncRNAs may be involved in biological processes similar to those regulated by their neighboring protein-coding genes (118). Thus, the biological function of the uncharacterized lncRNAs can be predicted based on the functionality of the nearby proteincoding genes. LncRNAs have been confirmed to participate in multiple processes, such as cell proliferation, development and diseases (119). LncRNAs are also involved in a variety of immune responses including inflammatory response and IFN-mediated response (120-122). However, most of the studies in the lncRNA field are limited to mammalian species. Currently, researchers are showing more interest in inquiring into the role of lncRNAs in teleost fish. Due to the development of high-throughput sequencing technology, the functional role of IncRNAs in fish immune response against pathogen infection has been gradually disclosed.

Previously, Boltaña et al. (123) reported the transcriptomic regulation of lncRNAs in Atlantic salmon during infectious salmon anemia virus (ISAV) infection. A total of 5,636 putative lncRNAs were identified, most of which were regulated in response to ISAV infection. Intriguingly, most of the lncRNAs 
were similarly regulated in response to ISAV infection and were enriched in genes correlated with innate immunity and antigen presentation. Remarkably, seven lncRNAs (Ss_lncRNA_575, Ss_lncRNA_1421, Ss_lncRNA_1969, Ss_lncRNA_2198, Ss_lncRNA_2753, Ss_lnRNA_4968 and Ss_lncRNA_4977) were strongly associated with antiviral mRNA expression. The expression of Ss_lncRNA_2198 was highly correlated with grass carp reovirus (GCRV)-induced gene 2 (GIG2) (124). Taken together, these results demonstrated that lncRNAs might be implicated in host defense response to ISAV infection. This study would be helpful for fully uncovering lncRNA regulation during ISAV pathogenesis. LncRNAs also affect the activity of miRNAs. The deregulated lncRNAs, miRNAs, and their target genes might constitute complex modulatory networks in the defense response to ISAV infection. Mechanistic characterization of the lncRNA/miRNA/mRNA axes will be beneficial to expand our understanding of viral pathogenesis in teleost fish. C. Gallardo-Escárate' research group previously revealed the ncRNA response during Piscirickettsia salmonis infection in Atlantic salmon (125). A total of 918 putative lncRNAs were identified, 425 of which were newly characterized for Atlantic salmon. These lncRNAs were strongly correlated with clathrinmediated endocytosis and iron homeostasis, demonstrating a potential mechanism of immune evasion by $P$. salmonis. This research group further profiled a widespread differential expression of lncRNAs in Atlantic salmon infected with different pathogens including ISAV, $P$. salmonis and the ectoparasite copepod Caligus rogercresseyi by comparative transcriptome analysis (126). The result showed that lncRNAs were widely regulated during pathogen infection, and this regulation was pathogen-specific and tightly associated with immune-related genes involved in innate immunity. LncRNAs have a broader spectrum of regulation, and serve as versatile regulators of gene expression (127). Intriguingly, a single lncRNA can mediate both the activation and suppression of immune responses (120). Both positive and negative transcriptional association between lncRNAs and their target genes could be expected in teleost fish. Further functional analyses are required to fully characterize the regulation of lncRNAs in the transcriptional responses of Atlantic salmon to invading pathogens.

A comprehensive study was previously performed to identify putative lncRNAs in the rainbow trout genome (128). A total of 54,503 putative lncRNAs were discovered. Of the putative lncRNAs, 2,935 lncRNAs displayed tissue-specific expression pattern. Moreover, the functional prediction indicated that several lncRNAs were positively associated with NF$\kappa \mathrm{B}$ inhibitor-like protein and MAPK1. However, the detailed mechanism underlying the regulation of genes by lncRNAs requires to be further surveyed. Moreover, a total of 9,674 large intergenic ncRNAs (lincRNAs) were previously found in rainbow trout based on the analysis of RNA-sequencing data (13). The predicted rainbow trout lincRNAs shared similar genomic features with lincRNAs from other species, such as human, mouse, and zebrafish. The lincRNAs exhibited tissuespecific expression pattern and were typically co-expressed with their adjoining genes. By clustering associated genes together, 34 co-expression gene modules consisting of 2,963
lincRNAs and 10,321 protein-coding genes were identified. Notably, 6 of 34 modules were associated with immune responses. LincRNAs in the 6 modules were co-expressed with genes implicated in $\mathrm{T}$ cell receptor signaling, immune cell activation, and antigen processing and presentation. The expression profile of lncRNAs in the intestine of rainbow trout was also analyzed, and hundreds of lncRNAs were differentially expressed in trout fed with functional diets based on pre- and probiotics compared to the control group (129). Some differentially expressed lncRNAs might regulate the expression of immune-related genes including TLR and TNF$\alpha$.

The expression profile of lncRNA was previously determined between rainbow trout strains, one a control strain and the other a strain fed an all plant-protein diet (130). A total of 142 unique antisense lncRNAs were found to be differentially expressed between the strains, 60 of which overlapped with genes involved in lipid metabolism and immune functionality, such as sterol regulatory element binding protein 1, sterol carrier protein 2, MHC class I heavy chain precursor, complement components $\mathrm{C} 3$ and $\mathrm{C} 9$, and IFN inducible protein 2. The differentially expressed lncRNAs in response to Flavobacterium psychrophilum challenge were analyzed in three genetic lines of rainbow trout (131). As a result, 556 differentially expressed lncRNAs were identified. Some differentially expressed lncRNAs were found to be strongly correlated with immune-relevant genes including complement components, and cytokines and chemokines. Several differentially expressed lncRNAs were associated with signaling molecules implicated in microbial infection processes, mTOR signaling pathway, T cell immune response and others. This study indicated that lncRNAs were able to control anti-bacterial immune responses in rainbow trout. A catalog of lncRNAs in the large yellow croaker has been discovered by characterization of the transcriptome in spleen, muscle, liver, and egg tissues (132). Accordingly, a total of 210 lincRNAs were identified in $V$. anguillarum-infected spleen tissue of the large yellow croaker. These lincRNAs were predicted to be engaged in immune responses, such as positive regulation of leukocyte/lymphocyte and T-cell activation. Specifically, two lincRNAs, Linc_08129.1 and Linc_32654.1, had the potential to activate the TLR signaling pathway to elicit host antiviral responses. The expression of four lincRNAs, Linc_00388.1, Linc_01738.1, Linc_06498.1, and Linc_09316.1, was elevated at $24 \mathrm{~h}$ after $V$. anguillarum infection and was then decreased at $48 \mathrm{~h}$. This result implied that the large yellow croaker lincRNAs tended to participate in the early stage of host immune responses. The depth of RNA sequencing used in this study might hamper the discovery of lncRNAs with low abundance. Further identification of lncRNAs in the large yellow croaker is required.

Low sequence conservation of lncRNAs has limited the elucidation of lncRNA function based on comparative sequence analysis. Further study on the structural and regulatory elements of lncRNAs would aid the identification and characterization of lncRNAs in teleost fish. Currently, the identification of lncRNAs largely depends on the bioinformatics analysis. The computational tools might mis-identify the lncRNAs harboring 
short conserved open reading frame (ORF) regions as proteincoding transcripts. Conversely, protein-coding transcripts containing short conserved ORFs might be mis-identified as lncRNAs. Ribosome profiling can be employed to assess the protein-coding characteristics of putative lncRNAs (133). High-throughput RNA sequencing and ribosome profiling could be applied to identify genuine lncRNAs in teleost fish.

LncRNAs have been shown to play central roles in mRNA processing, gene expression and epigenetic regulation (134). LncRNAs may represent a new layer of gene expression modulation responsible for controlling host immune responses against pathogen infection (135). More importantly, lncRNAs possess the potential to serve as a new class of diagnostic biomarkers for fish diseases. The recent discovery of immunerelated lncRNAs in teleost fish has opened up a new era of immune regulation exploration. Characterization of $\operatorname{lncRNAs}$ correlated with host defense responses would have deep impacts in the field of fish immunology. Functional crosstalk between IncRNAs and miRNAs in fish immune regulation is an important area of interest. Determining the roles of immune-related lncRNAs in teleost fish will facilitate our comprehension of their functional roles in immune responses. In-depth investigations on lncRNA regulation and function are warranted to comprehensively reveal the mechanism underlying modulation of fish immune responses by lncRNAs.

\section{Identification of circRNAs in Teleost Fish}

Circular RNAs (circRNAs), a novel type of ncRNAs, are characterized by covalently closed loop structures with neither $5^{\prime}$ end caps nor $3^{\prime}$ poly (A) tails (136). CircRNAs are evolutionarily conserved across multiple species, and display tissue-specific and development-dependent expression patterns (137). The function of circRNAs has become a research hotspot in recent years. Emerging evidence indicates that circRNAs are master regulators of gene expression by acting as miRNA sponges and perform important roles in a variety of biological processes (138140). CircRNAs also fulfill regulatory function in host immune responses $(141,142)$. Increasing transcriptome and phenotype data enable us to explore the biological function of circRNAs in teleost fish.

The identification of circRNAs from RNA-sequencing data in the large yellow croaker has been reported (143). A total of 975 circRNAs were detected, and the circRNA-host genes were enriched in translational progression, binding-related functions, RNA degradation and transport, and metabolic pathways. Intriguingly, KEGG enrichment showed that the circRNA-host genes were significantly enriched in complement and coagulation cascades, and antigen processing and presentation. The circRNAhost genes had at least two different circRNA isoforms, demonstrating that circRNAs had alternative circulations in the large yellow croaker. The regulation and formation mechanisms for alternative circularization of circRNAs in the large yellow croaker may be complex and need systematic study in the future. In addition, a number of circRNAs were predicted to harbor more than one miRNA binding sites. These circRNAs might have a broad impact in immune regulation by sponging miRNAs in the large yellow croaker. This study provided a valuable reference for circRNA study in teleost fish. Deep illumina sequencing was performed to discover the circRNAs in GCRV-infected grass carp (144). As a result, 5,052 novel circRNAs were identified in grass carp in response to viral infection. Moreover, 41 of the identified circRNAs were differentially expressed. Further analysis showed that 72 miRNAs were predicted to interact with the differentially expressed circRNAs. Target genes of the binding miRNAs mainly took part in immune response, hemostasis, blood coagulation, and complement and coagulation cascades. These results demonstrated that the circRNA-miRNA-mRNA axes existed in virally infected grass carp, providing novel insights into the molecular mechanism underlying pathogen infection and fish immune regulation. CircRNAs in zebrafish were sequenced and identified using the high-throughput sequencing technology combined with bioinformatics approach (145). A total of 3,868 circRNAs were found in zebrafish, 1,122 of which exhibited homology with human, mouse and coelacanth circRNAs. Accordingly, study on teleost circRNAs would also accelerate our efforts to fully elucidate circRNA-mediated regulatory networks in human owing to high conservation of circRNAs.

These studies provided valuable genetic resources for basic research and aquaculture improvement of teleost fish. The functional roles of circRNAs are receiving increasing attention. CircRNAs played an important role in antiviral immunity (146). It is likely that circRNAs function as pivotal regulators in fish defense system. However, few studies on circRNAs have been reported in teleost fish. More studies are needed to mine and characterize circRNAs in fish species. The detailed exploration of circRNA functions would shape our understanding of circRNA biology in teleost fish.

\section{CONCLUDING REMARKS}

In recent years, great progress has been made in elucidating the function of ncRNAs in fish immune responses which would benefit the rapid expanding aquaculture industry worldwide. Identification of immune-related ncRNAs in teleost fish is mainly based on the high-throughput sequencing technology. Highthroughput sequencing is a powerful platform for the genomewide identification of novel ncRNAs. The number of sequencing reads that are mapped to a particular ncRNA directly reflects the abundance of this ncRNA, indicating that high-throughput sequencing data can indicate the relative expression of identified ncRNAs. Thus, high-throughput sequencing results can be used for quantitative analysis of ncRNAs. Based on these clues, highthroughput sequencing platform seems to be the best choice for comprehensive profiling of ncRNAs in teleost fish. Besides, online resources and bioinformatics analysis are essential for the study on ncRNA biology and function.

A number of miRNAs have been found to control host immune responses by targeting immune-relevant genes in teleost fish. The explicit roles of miRNA/mRNA regulatory axes in fish defense responses need further exploration. In addition, lncRNAs and circRNAs have been confirmed to 
offset miRNA-mediated gene silencing by acting as ceRNAs. It will be intriguing to investigate the functional roles of lncRNA/miRNA/mRNA or circRNA/miRNA/mRNA regulatory networks in immune regulation. Further study on the biology of teleost ncRNAs will contribute to our understanding of the complex interaction between the regulatory ncRNAs and fish immune system during the process of pathogen infection.

Nevertheless, there is a lack of high quality reference genome/transcriptome resources for teleost fish, which restricts the ncRNA research in teleost fish. Meanwhile, the miRNAomes of fish species are incomplete. The identification and functional characterization of ncRNAs are crucial to increase our systematical understanding of the biological processes underlying teleost development, physiology and immunity. Moreover, investigations on teleost genetic regulatory elements, including miRNAs, lncRNAs, and circRNAs, would provide new insights into human gene regulatory networks through orthologous gene functional studies. Furthermore, increasing evidence suggests that ncRNAs hold great promise to be used as novel biomarkers for the control and treatment of fish diseases. Therefore, more research has to be focused on teleost ncRNAs which would accelerate the development of diagnostic markers

\section{REFERENCES}

1. Bizuayehu TT, Babiak I. MicroRNA in teleost fish. Genome Biol Evol. (2014) 6:1911-37. doi: 10.1093/gbe/evu151

2. Volff JN. Genome evolution and biodiversity in teleost fish. Heredity (2005) 94:280-94. doi: 10.1038/sj.hdy.6800635

3. Hurley IA, Mueller RL, Dunn KA, Schmidt EJ, Friedman M, Ho RK, et al. A new time-scale for ray-finned fish evolution. Proc Biol Sci. (2007) 274:489-98. doi: $10.1098 /$ rspb.2006.3749

4. Alexander RP, Fang G, Rozowsky J, Snyder M, Gerstein MB. Annotating non-coding regions of the genome. Nat Rev Genet. (2010) 11:559-71. doi: $10.1038 / \mathrm{nrg} 2814$

5. He L, Hannon GJ. MicroRNAs: small RNAs with a big role in gene regulation. Nat Rev Genet. (2004) 5:522-31. doi: 10.1038/nrg1379

6. Mercer TR, Dinger ME, Mattick JS. Long non-coding RNAs: insights into functions. Nat Rev Genet. (2009) 10:155-9. doi: 10.1038/nrg2521

7. Mercer TR, Mattick JS. Structure and function of long noncoding RNAs in epigenetic regulation. Nat Struct Mol Biol. (2013) 20:300-7. doi: $10.1038 / \mathrm{nsmb} .2480$

8. Takacs CM, Giraldez AJ. MicroRNAs as genetic sculptors: fishing for clues. Semin Cell Dev Biol. (2010) 21:760-7. doi: 10.1016/j.semcdb.2010.02.003

9. Bizuayehu TT, Johansen SD, Puvanendran V, Toften H, Babiak I. Temperature during early development has long-term effects on microRNA expression in Atlantic cod. BMC Genomics (2015) 16:305. doi: 10.1186/s12864-015-1503-7

10. Andreassen R, Rangnes F, Sivertsen M, Chiang M, Tran M, Worren MM. Discovery of miRNAs and their corresponding miRNA genes in Atlantic Cod (Gadus morhua): use of stable miRNAs as reference genes reveals subgroups of miRNAs that are highly expressed in particular organs. PLoS ONE (2016) 11:e0153324. doi: 10.1371/journal.pone.0153324

11. Mennigen JA. Micromanaging metabolism-a role for miRNAs in teleost energy metabolism. Comp Biochem Physiol B Biochem Mol Biol. (2016) 199:115-25. doi: 10.1016/j.cbpb.2015.09.001

12. Basu S, Hadzhiev Y, Petrosino G, Nepal C, Gehrig J, Armant O, et al. The Tetraodon nigroviridis reference transcriptome: developmental transition, length retention and microsynteny of long non-coding RNAs in a compact vertebrate genome. Sci Rep. (2016) 6:33210. doi: 10.1038/srep33210 and therapeutic targets for pathogenic diseases in economically important teleost fish species.

So far, a variety of ncRNAs have been identified in teleost fish through the high-throughput sequencing technology. Target genes of these teleost ncRNAs are predicted through bioinformatics analysis. Therefore, functional validation using experimental approaches will be the next important step in the research on ncRNAs associated with immune responses in teleost fish. With the growing number of available sequenced genomes, the functional roles of ncRNAs in fish immune responses will be better understood in the future.

\section{AUTHOR CONTRIBUTIONS}

MW wrote the manuscript. SJ, WW, and FY prepared tables and figures. WC, PL, and $\mathrm{KW}$ edited the manuscript.

\section{FUNDING}

This work was supported by the National Natural Science Foundation of China (81701991) and Applied Basic Research Programs of Qingdao, China (17-1-1-59-jch).

13. Wang J, Fu L, Koganti PP, Wang L, Hand JM, Ma H, et al. Identification and functional prediction of large intergenic noncoding RNAs (lincRNAs) in rainbow trout (Oncorhynchus mykiss). Mar Biotechnol. (2016) 18:271-82. doi: 10.1007/s10126-016-9689-5

14. Eslamloo K, Inkpen SM, Rise ML, Andreassen R. Discovery of microRNAs associated with the antiviral immune response of Atlantic cod macrophages. Mol Immunol. (2018) 93:152-61. doi: 10.1016/j.molimm.2017. 11.015

15. Xu T, Chu Q, Cui J, Huo R. MicroRNA-216a inhibits NF-kappaB-mediated inflammatory cytokine production in teleost fish by modulating p65. Infect Immun. (2018) 86:e00256-18. doi: 10.1128/IAI.00256-18

16. Wong CM, Tsang FH, Ng IO. Non-coding RNAs in hepatocellular carcinoma: molecular functions and pathological implications. Nat Rev Gastroenterol Hepatol. (2018) 15:137-51. doi: 10.1038/nrgastro.2017.169

17. Gomes AQ, Nolasco S, Soares H. Non-coding RNAs: multitasking molecules in the cell. Int J Mol Sci. (2013) 14:16010-39. doi: 10.3390/ijms140816010

18. Cech TR, Steitz JA. The noncoding RNA revolution-trashing old rules to forge new ones. Cell (2014) 157:77-94. doi: 10.1016/j.cell.2014.03.008

19. Ambros V. The functions of animal microRNAs. Nature (2004) 431:350-5. doi: 10.1038 /nature 02871

20. Billerey C, Boussaha M, Esquerre D, Rebours E, Djari A, Meersseman C, et al. Identification of large intergenic non-coding RNAs in bovine muscle using next-generation transcriptomic sequencing. BMC Genomics (2014) 15:499. doi: 10.1186/1471-2164-15-499

21. Peschansky VJ, Wahlestedt C. Non-coding RNAs as direct and indirect modulators of epigenetic regulation. Epigenetics (2014) 9:3-12. doi: 10.4161/epi.27473

22. Zhang YC, Liao JY, Li ZY, Yu Y, Zhang JP, Li QF, et al. Genome-wide screening and functional analysis identify a large number of long noncoding RNAs involved in the sexual reproduction of rice. Genome Biol. (2014) 15:512. doi: 10.1186/s13059-014-0512-1

23. Mattick JS, Rinn JL. Discovery and annotation of long noncoding RNAs. Nat Struct Mol Biol. (2015) 22:5-7. doi: 10.1038/nsmb.2942

24. Memczak S, Jens M, Elefsinioti A, Torti F, Krueger J, Rybak A, et al. Circular RNAs are a large class of animal RNAs with regulatory potency. Nature (2013) 495:333-8. doi: 10.1038/nature11928 
25. Guo JU, Agarwal V, Guo H, Bartel DP. Expanded identification and characterization of mammalian circular RNAs. Genome Biol. (2014) 15:409. doi: 10.1186/s13059-014-0409-Z

26. Westholm JO, Miura P, Olson S, Shenker S, Joseph B, Sanfilippo P, et al. Genome-wide analysis of drosophila circular RNAs reveals their structural and sequence properties and age-dependent neural accumulation. Cell Rep. (2014) 9:1966-80. doi: 10.1016/j.celrep.2014.10.062

27. Ivanov A, Memczak S, Wyler E, Torti F, Porath HT, Orejuela MR, et al. Analysis of intron sequences reveals hallmarks of circular RNA biogenesis in animals. Cell Rep. (2015) 10:170-7. doi: 10.1016/j.celrep.2014.12.019

28. Sirotkin AV, Laukova M, Ovcharenko D, Brenaut P, Mlyncek M. Identification of microRNAs controlling human ovarian cell proliferation and apoptosis. J Cell Physiol. (2010) 223:49-56. doi: 10.1002/jcp.21999

29. Mohr AM, Mott JL. Overview of microRNA biology. Semin Liver Dis. (2015) 35:3-11. doi: 10.1055/s-0034-1397344

30. Valadkhan S. The role of snRNAs in spliceosomal catalysis. Prog Mol Biol Transl Sci. (2013) 120:195-228. doi: 10.1016/B978-0-12-381286-5.00006-8

31. Hang J, Wan R, Yan C, Shi Y. Structural basis of pre-mRNA splicing. Science (2015) 349:1191-8. doi: 10.1126/science.aac8159

32. Kiss-Laszlo Z, Henry Y, Bachellerie JP, Caizergues-Ferrer M, Kiss T. Sitespecific ribose methylation of preribosomal RNA: a novel function for small nucleolar RNAs. Cell (1996) 85:1077-88.

33. Ni J, Tien AL, Fournier MJ. Small nucleolar RNAs direct site-specific synthesis of pseudouridine in ribosomal RNA. Cell (1997) 89:565-73.

34. King TH, Liu B, McCully RR, Fournier MJ. Ribosome structure and activity are altered in cells lacking snoRNPs that form pseudouridines in the peptidyl transferase center. Mol Cell (2003) 11:425-35.

35. Tam OH, Aravin AA, Stein P, Girard A, Murchison EP, Cheloufi S, et al. Pseudogene-derived small interfering RNAs regulate gene expression in mouse oocytes. Nature (2008) 453:534-8. doi: 10.1038/nature06904

36. Carthew RW, Sontheimer EJ. Origins and mechanisms of miRNAs and siRNAs. Cell (2009) 136:642-55. doi: 10.1016/j.cell.2009.01.035

37. Guo Y, Bosompem A, Mohan S, Erdogan B, Ye F, Vickers KC, et al. Transfer RNA detection by small RNA deep sequencing and disease association with myelodysplastic syndromes. BMC Genomics (2015) 16:727. doi: 10.1186/s12864-015-1929-y

38. Koh CS, Sarin LP. Transfer RNA modification and infection - Implications for pathogenicity and host responses. Biochim Biophys Acta (2018) 1861:41932. doi: 10.1016/j.bbagrm.2018.01.015

39. Aravin AA, Hannon GJ, Brennecke J. The Piwi-piRNA pathway provides an adaptive defense in the transposon arms race. Science (2007) 318:761-4. doi: 10.1126/science.1146484

40. Aravin AA, Sachidanandam R, Girard A, Fejes-Toth K, Hannon GJ. Developmentally regulated piRNA clusters implicate MILI in transposon control. Science (2007) 316:744-7. doi: 10.1126/science.1142612

41. Thomson T, Lin $\mathrm{H}$. The biogenesis and function of PIWI proteins and piRNAs: progress and prospect. Annu Rev Cell Dev Biol. (2009) 25:355-76. doi: 10.1146/annurev.cellbio.24.110707.175327

42. Derrien T, Johnson R, Bussotti G, Tanzer A, Djebali S, Tilgner H, et al. The GENCODE v7 catalog of human long noncoding RNAs: analysis of their gene structure, evolution, and expression. Genome Res. (2012) 22:1775-89. doi: $10.1101 /$ gr.132159.111

43. Rinn JL, Chang HY. Genome regulation by long noncoding RNAs. Annu Rev Biochem. (2012) 81:145-66. doi: 10.1146/annurev-biochem-051410-092902

44. Flynn RA, Chang HY. Long noncoding RNAs in cell-fate programming and reprogramming. Cell Stem Cell (2014) 14:752-61. doi: 10.1016/j.stem.2014.05.014

45. Lasda E, Parker R. Circular RNAs: diversity of form and function. RNA (2014) 20:1829-42. doi: 10.1261/rna.047126.114

46. Muluhngwi P, Klinge CM. Roles for miRNAs in endocrine resistance in breast cancer. Endocr Relat Cancer (2015) 22:R279-300. doi: 10.1530/ERC-15-0355

47. Watanabe $\mathrm{T}$, Lin $\mathrm{H}$. Posttranscriptional regulation of gene expression by Piwi proteins and piRNAs. Mol Cell (2014) 56:18-27. doi: 10.1016/j.molcel.2014.09.012

48. Dykes IM, Emanueli C. Transcriptional and Post-transcriptional gene regulation by long non-coding RNA. Genomics Proteomics Bioinformatics (2017) 15:177-86. doi: 10.1016/j.gpb.2016.12.005
49. Hu YH, Zhang BC, Zhou HZ, Guan XL, Sun L. Edwardsiella tarda-induced miRNAs in a teleost host: global profile and role in bacterial infection as revealed by integrative miRNA-mRNA analysis. Virulence (2017) 8:1457-64. doi: 10.1080/21505594.2017.1356541

50. Chu Q, Gao Y, Bi D, Xu T. MicroRNA-148 as a negative regulator of the common TLR adaptor mediates inflammatory response in teleost fish. Sci Rep. (2017) 7:4124. doi: 10.1038/s41598-017-04354-9

51. Chu Q, Sun Y, Cui J, Xu T. Inducible microRNA-214 contributes to the suppression of NF-kappaB-mediated inflammatory response via targeting myd88 gene in fish. J Biol Chem. (2017) 292:5282-90. doi: 10.1074/jbc.M117.777078

52. Chu Q, Sun Y, Cui J, Xu T. MicroRNA-3570 Modulates the NF-kappaB pathway in teleost fish by targeting MyD88. J Immunol. (2017) 198:3274-82. doi: 10.4049/jimmunol.1602064

53. Xu T, Chu Q, Cui J, Bi D. Inducible MicroRNA-3570 feedback inhibits the RIG-I-dependent innate immune response to Rhabdovirus in teleost fish by targeting MAVS/IPS-1. J Virol. (2018) 92:e01594-17. doi: 10.1128/JVI.01594-17

54. Zhao X, Chu Q, Cui J, Xu T. microRNA-19a as a negative regulator in TLR signaling pathway by direct targeting myeloid differentiation factor 88 in miluy croaker. Dev Comp Immunol. (2018) 87:171-5. doi: 10.1016/j.dci.2018.06.009

55. Chu Q, Xu T. miR-192 targeting IL-1RI regulates the immune response in miiuy croaker after pathogen infection in vitro and in vivo. Fish Shellfish Immunol. (2016) 54:537-43. doi: 10.1016/j.fsi.2016.05.007

56. Xu T, Chu Q, Cui J, Zhao X. The inducible microRNA-203 in fish represses the inflammatory responses to Gram-negative bacteria by targeting IL-1 receptor-associated kinase 4. J Biol Chem. (2018) 293:1386-96. doi: 10.1074/jbc.RA117.000158

57. Sun Y, Chu Q, Zhao X, Zhou Z, Bi D, Xu T. microRNA-375 modulates the NF-kappaB pathway in miiuy croaker by targeting DUSP1 gene. Dev Comp Immunol. (2018) 86:196-202. doi: 10.1016/j.dci.2018.05.010

58. Ni S, Yan Y, Cui H, Yu Y, Huang Y, Qin Q. Fish miR-146a promotes Singapore grouper iridovirus infection by regulating cell apoptosis and NF-kappaB activation. J Gen Virol. (2017) 98:1489-99. doi: 10.1099/jgv.0. 000811

59. Sun Y, Han J, Chu Q, Liu X, Xu T. microRNA-210 participates in regulating RIG-I signaling pathway via targeting DUBA in miiuy croaker after poly(I:C) stimulation. Fish Shellfish Immunol. (2018) 77:1-7. doi: 10.1016/j.fsi.2018.02.003

60. Xu T, Chu Q, Cui J. Rhabdovirus-Inducible MicroRNA-210 modulates antiviral innate immune response via targeting STING/MITA in fish. $J$ Immunol. (2018) 201:982-94. doi: 10.4049/jimmunol.1800377

61. Zhang BC, Zhou ZJ, Sun L. pol-miR-731, a teleost miRNA upregulated by megalocytivirus, negatively regulates virus-induced type I interferon response, apoptosis, and cell cycle arrest. Sci Rep. (2016) 6:28354. doi: 10.1038/srep28354

62. Chan J, Hu X, Wang C, Xu Q. miRNA-152 targets GATA1 to regulate erythropoiesis in Chionodraco hamatus. Biochem Biophys Res Commun. (2018) 501:711-7. doi: 10.1016/j.bbrc.2018.05.053

63. Ni S, Yu Y, Wei J, Zhou L, Wei S, Yan Y, et al. MicroRNA-146a promotes red spotted grouper nervous necrosis virus (RGNNV) replication by targeting TRAF6 in orange spotted grouper, Epinephelus coioides. Fish Shellfish Immunol. (2018) 72:9-13. doi: 10.1016/j.fsi.2017.10.020

64. Valenzuela-Munoz V, Novoa B, Figueras A, Gallardo-Escarate C. Modulation of Atlantic salmon miRNome response to sea louse infestation. Dev Comp Immunol. (2017) 76:380-91. doi: 10.1016/j.dci.2017.07.009

65. Zhang C, Yi L, Feng S, Liu X, Su J, Lin L, et al. MicroRNA miR214 inhibits snakehead vesiculovirus replication by targeting the coding regions of viral N and P. J Gen Virol. (2017) 98:1611-9. doi: 10.1099/jgv.0. 000854

66. Cui J, Gao Y, Chu Q, Bi D, Xu T. miRNA-8159 is involved in TLR signaling pathway regulation after pathogen infection by direct targeting TLR13 in miiuy croaker. Fish Shellfish Immunol. (2017) 66:531-9. doi: 10.1016/j.fsi.2017.05.046

67. Chu Q, Sun Y, Bi D, Cui J, Xu T. Up-regulated of miR-8159-5p and miR-217$5 \mathrm{p}$ by LPS stimulation negatively co-regulate TLR1 in miiuy croaker. Dev Comp Immunol. (2017) 67:117-25. doi: 10.1016/j.dci.2016.11.004 
68. Wang $\mathrm{Y}, \mathrm{Xu}$ G, Han J, Xu T. miR-200a-3p regulates TLR1 expression in bacterial challenged miiuy croaker. Dev Comp Immunol. (2016) 63:181-6. doi: 10.1016/j.dci.2016.06.004

69. Cui J, Chu Q, Xu T. miR-122 involved in the regulation of tolllike receptor signaling pathway after Vibrio anguillarum infection by targeting TLR14 in miiuy croaker. Fish Shellfish Immunol. (2016) 58:67-72. doi: 10.1016/j.fsi.2016.09.027

70. Bi D, Cui J, Chu Q, Xu T. MicroRNA-21 contributes to suppress cytokines production by targeting TLR28 in teleost fish. Mol Immunol. (2017) 83:10714. doi: 10.1016/j.molimm.2017.01.016

71. Xu XY, Shen YB, Fu JJ, Yu HY, Huang WJ, Lu LQ, et al. MicroRNA-induced negative regulation of TLR-5 in grass carp, Ctenopharyngodon idella. Sci Rep. (2016) 6:18595. doi: 10.1038/srep18595

72. Najib A, Kim MS, Choi SH, Kang YJ, Kim KH. Changes in microRNAs expression profile of olive flounder (Paralichthys olivaceus) in response to viral hemorrhagic septicemia virus (VHSV) infection. Fish Shellfish Immunol. (2016) 51:384-91. doi: 10.1016/j.fsi.2016.03.014

73. Andreassen R, Hoyheim B. miRNAs associated with immune response in teleost fish. Dev Comp Immunol. (2017) 75:77-85. doi: 10.1016/j.dci.2017.02.023

74. Andreassen R, Woldemariam NT, Egeland IO, Agafonov O, Sindre H, Hoyheim B. Identification of differentially expressed Atlantic salmon miRNAs responding to salmonid alphavirus (SAV) infection. BMC Genomics (2017) 18:349. doi: 10.1186/s12864-017-3741-3

75. Tapiovaara H, Olesen NJ, Linden J, Rimaila-Parnanen E, von Bonsdorff CH. Isolation of an iridovirus from pike-perch Stizostedion lucioperca. Dis Aquat Organ. (1998) 32:185-93. doi: 10.3354/dao032185

76. Ariel E, Jensen BB. Challenge studies of European stocks of redfin perch, Perca fluviatilis L., and rainbow trout, Oncorhynchus mykiss (Walbaum), with epizootic haematopoietic necrosis virus. J Fish Dis. (2009) 32:1017-25. doi: 10.1111/j.1365-2761.2009.01088.x

77. Shi CY, Jia KT, Yang B, Huang J. Complete genome sequence of a Megalocytivirus (family Iridoviridae) associated with turbot mortality in China. Virol J. (2010) 7:159. doi: 10.1186/1743-422X-7-159

78. Zhang BC, Zhang J, Sun L. In-depth profiling and analysis of host and viral microRNAs in Japanese flounder (Paralichthys olivaceus) infected with megalocytivirus reveal involvement of microRNAs in host-virus interaction in teleost fish. BMC Genomics (2014) 15:878. doi: 10.1186/1471-216415-878

79. Everett H, McFadden G. Apoptosis: an innate immune response to virus infection. Trends Microbiol. (1999) 7:160-5.

80. Itoh H, Ogura M, Komatsuda A, Wakui H, Miura AB, Tashima Y. A novel chaperone-activity-reducing mechanism of the $90-\mathrm{kDa}$ molecular chaperone HSP90. Biochem J. (1999) 343:697-703.

81. Connor JH, McKenzie MO, Parks GD, Lyles DS. Antiviral activity and RNA polymerase degradation following Hsp90 inhibition in a range of negative strand viruses. Virology (2007) 362:109-19. doi: 10.1016/j.virol.2006. 12.026

82. Chase G, Deng T, Fodor E, Leung BW, Mayer D, Schwemmle M, et al. Hsp90 inhibitors reduce influenza virus replication in cell culture. Virology (2008) 377:431-9. doi: 10.1016/j.virol.2008.04.040

83. Guo C, Cui H, Ni S, Yan Y, Qin Q. Comprehensive identification and profiling of host miRNAs in response to Singapore grouper iridovirus (SGIV) infection in grouper (Epinephelus coioides). Dev Comp Immunol. (2015) 52:226-35. doi: 10.1016/j.dci.2015.05.014

84. Mehinto AC, Martyniuk CJ, Spade DJ, Denslow ND. Applications for nextgeneration sequencing in fish ecotoxicogenomics. Front Genet. (2012) 3:62. doi: 10.3389/fgene.2012.00062

85. Liu X, Tu J, Yuan J, Liu X, Zhao L, Dawar FU, et al. Identification and characterization of micrornas in Snakehead fish cell line upon snakehead fish Vesiculovirus Infection. Int J Mol Sci. (2016) 17:154. doi: 10.3390/ijms17020154

86. Schyth BD, Bela-Ong DB, Jalali SA, Kristensen LB, Einer-Jensen K, Pedersen FS, et al. Two virus-induced MicroRNAs known only from teleost fishes are orthologues of MicroRNAs involved in cell cycle control in humans. PLoS ONE (2015) 10:e0132434. doi: 10.1371/journal.pone.0132434

87. Bela-ong DB, Schyth BD, Zou J, Secombes C. J, Lorenzen N. (2015). Involvement of two microRNAs in the early immune response to
DNA vaccination against a fish rhabdovirus. Vaccine 33, 3215-22. doi: 10.1016/j.vaccine.2015.04.092

88. Wu S, Liu L, Zohaib A, Lin L, Yuan J, Wang M, et al. MicroRNA profile analysis of Epithelioma papulosum cyprini cell line before and after SVCV infection. Dev Comp Immunol. (2015) 48:124-8. doi: 10.1016/j.dci.2014.09.012

89. Han J, $\mathrm{Xu} \mathrm{G}, \mathrm{Xu}$ T. The miiuy croaker microRNA transcriptome and microRNA regulation of RIG-I like receptor signaling pathway after poly(I:C) stimulation. Fish Shellfish Immunol. (2016) 54:419-26. doi: 10.1016/j.fsi.2016.04.126

90. Chaudhary PM, Eby MT, Jasmin A, Kumar A, Liu L, Hood L. Activation of the NF-kappaB pathway by caspase 8 and its homologs. Oncogene (2000) 19:4451-60. doi: 10.1038/sj.onc.1203812

91. Seth RB, Sun L, Ea CK, Chen ZJ. Identification and characterization of MAVS, a mitochondrial antiviral signaling protein that activates NFkappaB and IRF 3. Cell (2005) 122:669-82. doi: 10.1016/j.cell.2005. 08.012

92. Mikkelsen SS, Jensen SB, Chiliveru S, Melchjorsen J, Julkunen I, Gaestel M, et al. RIG-I-mediated activation of p38 MAPK is essential for viral induction of interferon and activation of dendritic cells: dependence on TRAF2 and TAK1. J Biol Chem. (2009) 284:10774-82. doi: 10.1074/jbc.M8072 72200

93. Yan Y, Cui H, Jiang S, Huang Y, Huang X, Wei S, et al. Identification of a novel marine fish virus, Singapore grouper iridovirus-encoded microRNAs expressed in grouper cells by Solexa sequencing. PLoS ONE (2011) 6:e19148. doi: 10.1371/journal.pone.0019148

94. Donohoe $\mathrm{OH}$, Henshilwood K, Way K, Hakimjavadi R, Stone DM, Walls D. Identification and characterization of Cyprinid Herpesvirus3 (CyHV-3) encoded MicroRNAs. PLoS ONE (2015) 10:e125434. doi: 10.1371/journal.pone.0125434

95. Penades JR, Donderis J, Garcia-Caballer M, Tormo-Mas MA, Marina A. dUTPases, the unexplored family of signalling molecules. Curr Opin Microbiol. (2013) 16:163-70. doi: 10.1016/j.mib.2013.02.005

96. Yan Y, Guo C, Ni S, Wei J, Li P, Wei S, et al. Singapore grouper iridovirus (SGIV) encoded SGIV-miR-13 attenuates viral infection via modulating major capsid protein expression. Virus Res. (2015) 205:45-53. doi: 10.1016/j.virusres.2015.05.010

97. Thomson BJ. Viruses and apoptosis. Int J Exp Pathol. (2001) 82:65-76. doi: 10.1111/j.1365-2613.2001.iep0082-0065-X

98. Guo C, Yan Y, Cui H, Huang X, Qin Q. miR-homoHSV of Singapore grouper iridovirus (SGIV) inhibits expression of the SGIV pro-apoptotic factor LITAF and attenuates cell death. PLoS ONE (2013) 8:e83027. doi: 10.1371/journal.pone.0083027

99. Yuhong J, Leilei T, Fuyun Z, Hongyang J, Xiaowen L, Liying Y, et al. Identification and characterization of immune-related microRNAs in blunt snout bream, Megalobrama amblycephala. Fish Shellfish Immunol. (2016) 49:470-92. doi: 10.1016/j.fsi.2015.12.013

100. Ordas A, Kanwal Z, Lindenberg V, Rougeot J, Mink M, Spaink HP, et al. MicroRNA-146 function in the innate immune transcriptome response of zebrafish embryos to Salmonella typhimurium infection. BMC Genomics (2013) 14:696. doi: 10.1186/1471-2164-14-696

101. Zhao L, Lu H, Meng Q, Wang J, Wang W, Yang L, et al. Profilings of MicroRNAs in the liver of common carp (Cyprinus carpio) infected with flavobacterium columnare. Int J Mol Sci. (2016) 17:566. doi: 10.3390/ijms17040566

102. Sha Z, Gong G, Wang S, Lu Y, Wang L, Wang Q, et al. Identification and characterization of Cynoglossus semilaevis microRNA response to Vibrio anguillarum infection through high-throughput sequencing. Dev Comp Immunol. (2014) 44:59-69. doi: 10.1016/j.dci.2013. 11.014

103. Wang B, Gan Z, Cai S, Wang Z, Yu D, Lin Z, et al. Comprehensive identification and profiling of Nile tilapia (Oreochromis niloticus) microRNAs response to Streptococcus agalactiae infection through high-throughput sequencing. Fish Shellfish Immunol. (2016) 54:93-106. doi: 10.1016/j.fsi.2016.03.159

104. Rajaram MV, Ni B, Morris JD, Brooks MN, Carlson TK, Bakthavachalu B, et al. Mycobacterium tuberculosis lipomannan blocks TNF biosynthesis by regulating macrophage MAPK-activated protein kinase 2 (MK2) and 
microRNA miR-125b. Proc Natl Acad Sci USA. (2011) 108:17408-13. doi: 10.1073/pnas.1112660108

105. Xu G, Han J, Xu T. Comparative analysis of the small RNA transcriptomes of miiuy croaker revealed microRNA-mediated regulation of TLR signaling pathway response to Vibrio anguillarum infection. Fish Shellfish Immunol. (2016) 52:248-57. doi: 10.1016/j.fsi.2016.03.011

106. Gong G, Sha Z, Chen S, Li C, Yan H, Chen Y, et al. Expression profiling analysis of the microRNA response of Cynoglossus semilaevis to Vibrio anguillarum and other stimuli. Mar Biotechnol. (2015) 17:338-52. doi: $10.1007 / \mathrm{s} 10126-015-9623-2$

107. Jia K, Thomas C, Akbar M, Sun Q, Adams-Huet B, Gilpin C, et al. Autophagy genes protect against Salmonella typhimurium infection and mediate insulin signaling-regulated pathogen resistance. Proc Natl Acad Sci USA. (2009) 106:14564-9. doi: 10.1073/pnas.0813319106

108. Jo EK, Yuk JM, Shin DM, Sasakawa C. Roles of autophagy in elimination of intracellular bacterial pathogens. Front Immunol. (2013) 4:97. doi: 10.3389/fimmu.2013.00097

109. Qiang J, Tao F, He J, Sun L, Xu P, Bao W. Effects of exposure to Streptococcus iniae on microRNA expression in the head kidney of genetically improved farmed tilapia (Oreochromis niloticus). BMC Genomics (2017) 18:190. doi: 10.1186/s12864-017-3591-z

110. Mogensen TH. Pathogen recognition and inflammatory signaling in innate immune defenses. Clin Microbiol Rev. (2009) 22:240-73. doi: 10.1128/CMR.00046-08

111. Li Y, Song X, Wang W, Wang L, Yi Q, Jiang S, et al. The hematopoiesis in gill and its role in the immune response of Pacific oyster Crassostrea gigas against secondary challenge with Vibrio splendidus. Dev Comp Immunol. (2017). 71:59-69. doi: 10.1016/j.dci.2017.01.024

112. Netea MG, van der Graaf C, Van der Meer JW, Kullberg BJ. Tolllike receptors and the host defense against microbial pathogens: bringing specificity to the innate-immune system. J Leukoc Biol. (2004) 75:749-55. doi: $10.1189 / \mathrm{jlb} .1103543$

113. Pietretti D, Wiegertjes GF. Ligand specificities of Toll-like receptors in fish: indications from infection studies. Dev Comp Immunol. (2014) 43:205-22. doi: 10.1016/j.dci.2013.08.010

114. Zhang J, Kong X, Zhou C, Li L, Nie G, Li X. Toll-like receptor recognition of bacteria in fish: ligand specificity and signal pathways. Fish Shellfish Immunol. (2014) 41:380-8. doi: 10.1016/j.fsi.2014. 09.022

115. Wang Y, Li J, Han J, Shu C, Xu T. Identification and characteristic analysis of TLR28: a novel member of the TLR1 family in teleost. Dev Comp Immunol. (2016) 62:102-7. doi: 10.1016/j.dci.2016.05.001

116. Cabili MN, Trapnell C, Goff L, Koziol M, Tazon-Vega B, Regev A, et al. Integrative annotation of human large intergenic noncoding RNAs reveals global properties and specific subclasses. Genes Dev. (2011) 25:1915-27. doi: $10.1101 /$ gad.17446611

117. Guil S, Esteller M. Cis-acting noncoding RNAs: friends and foes. Nat Struct Mol Biol. (2012) 19:1068-75. doi: 10.1038/nsmb.2428

118. Luo S, Lu JY, Liu L, Yin Y, Chen C, Han X, et al. Divergent lncRNAs regulate gene expression and lineage differentiation in pluripotent cells. Cell Stem Cell. (2016) 18:637-52. doi: 10.1016/j.stem.2016.01.024

119. Peng X, Gralinski L, Armour CD, Ferris MT, Thomas MJ, Proll S, et al. Unique signatures of long noncoding RNA expression in response to virus infection and altered innate immune signaling. MBio (2010) 1:e00206-10. doi: 10.1128/mBio.00206-10

120. Carpenter S, Aiello D, Atianand MK, Ricci EP, Gandhi P, Hall LL, et al. A long noncoding RNA mediates both activation and repression of immune response genes. Science (2013) 341:789-92. doi: 10.1126/science. 1240925

121. Hu G, Tang Q, Sharma S, Yu F, Escobar TM, Muljo SA, et al. Expression and regulation of intergenic long noncoding RNAs during $\mathrm{T}$ cell development and differentiation. Nat Immunol. (2013) 14:1190-8. doi: 10.1038/ ni. 2712

122. Kambara H, Niazi F, Kostadinova L, Moonka DK, Siegel CT, Post AB, et al. Negative regulation of the interferon response by an interferoninduced long non-coding RNA. Nucleic Acids Res. (2014) 42:10668-80. doi: $10.1093 /$ nar/gku713
123. Boltana S, Valenzuela-Miranda D, Aguilar A, Mackenzie S, GallardoEscarate C. Long noncoding RNAs (lncRNAs) dynamics evidence immunomodulation during ISAV-Infected Atlantic salmon (Salmo salar). Sci Rep. (2016) 6:22698. doi: 10.1038/srep22698

124. Zhang YB, Liu TK, Jiang J, Shi J, Liu Y, Li S, et al. Identification of a novel Gig2 gene family specific to non-amniote vertebrates. PLoS ONE (2013) 8:e60588. doi: 10.1371/journal.pone. 0060588

125. Valenzuela-Miranda D, Gallardo-Escarate C. Novel insights into the response of Atlantic salmon (Salmo salar) to Piscirickettsia salmonis: interplay of coding genes and lncRNAs during bacterial infection. Fish Shellfish Immunol. (2016) 59:427-38. doi: 10.1016/j.fsi.2016.11.001

126. Tarifeno-Saldivia E, Valenzuela-Miranda D, Gallardo-Escarate C. In the shadow: The emerging role of long non-coding RNAs in the immune response of Atlantic salmon. Dev Comp Immunol. (2017) 73:193-205. doi: 10.1016/j.dci.2017.03.024

127. Nie L, Wu HJ, Hsu JM, Chang SS, Labaff AM, Li CW, et al. Long non-coding RNAs: versatile master regulators of gene expression and crucial players in cancer. Am J Transl Res. (2012) 4:127-50.

128. Al-Tobasei R, Paneru B, Salem M. Genome-wide discovery of long non-coding RNAs in rainbow trout. PLoS ONE (2016) 11:e0148940. doi: 10.1371 /journal.pone. 0148940

129. Nunez-Acuna G, Detree C, Gallardo-Escarate C, Goncalves AT. Functional diets modulate lncRNA-coding RNAs and gene interactions in the intestine of rainbow trout oncorhynchus mykiss. Mar Biotechnol. (2017) 19:287-300. doi: $10.1007 / \mathrm{s} 10126-017-9750-\mathrm{z}$

130. Abernathy J, Overturf K. Expression of antisense long noncoding RNAs as potential regulators in rainbow trout with different tolerance to plant-based diets. Anim Biotechnol. doi: 10.1080/10495398.2017.1401546. [Epub ahead of print].

131. Paneru B, Al-Tobasei R, Palti Y, Wiens GD, Salem M. Differential expression of long non-coding RNAs in three genetic lines of rainbow trout in response to infection with Flavobacterium psychrophilum. Sci Rep. (2016) 6:36032. doi: $10.1038 /$ srep36032

132. Jiang L, Liu W, Zhu A, Zhang J, Zhou J, Wu C. Transcriptome analysis demonstrate widespread differential expression of long noncoding RNAs involve in Larimichthys crocea immune response. Fish Shellfish Immunol. (2016) 51:1-8. doi: 10.1016/j.fsi.2016.02.001

133. Ingolia NT, Brar GA, Stern-Ginossar N, Harris MS, Talhouarne GJ, Jackson SE, et al. Ribosome profiling reveals pervasive translation outside of annotated protein-coding genes. Cell Rep. (2014) 8:1365-79. doi: 10.1016/j.celrep.2014.07.045

134. Ahmed W, Liu ZF. Long non-coding RNAs: novel players in regulation of immune response upon Herpesvirus infection. Front Immunol. (2018) 9:761. doi: $10.3389 /$ fimmu.2018.00761

135. Chen YG, Satpathy AT, Chang HY. Gene regulation in the immune system by long noncoding RNAs. Nat Immunol. (2017) 18:962-72. doi: 10.1038/ni.3771

136. Jeck WR, Sharpless NE. Detecting and characterizing circular RNAs. Nat Biotechnol. (2014) 32:453-61. doi: 10.1038/nbt.2890

137. Barrett SP, Salzman J. Circular RNAs: analysis, expression and potential functions. Development (2016) 143:1838-47. doi: 10.1242/dev.128074

138. Kulcheski FR, Christoff AP, Margis R. Circular RNAs are miRNA sponges and can be used as a new class of biomarker. J Biotechnol. (2016) 238:42-51. doi: 10.1016/j.jbiotec.2016.09.011

139. Huang S, Yang B, Chen BJ, Bliim N, Ueberham U, Arendt T, et al. The emerging role of circular RNAs in transcriptome regulation. Genomics (2017) 109:401-7. doi: 10.1016/j.ygeno.2017.06.005

140. Meng X, Li X, Zhang P, Wang J, Zhou Y, Chen M. Circular RNA: an emerging key player in RNA world. Brief Bioinformatics (2017) 18:547-57. doi: 10.1093/bib/bbw045

141. Chen YG, Kim MV, Chen X, Batista PJ, Aoyama S, Wilusz JE, et al. Sensing self and foreign circular RNAs by intron identity. Mol Cell (2017) 67:228-38 e225. doi: 10.1016/j.molcel.2017.05.022

142. Li X, Liu CX, Xue W, Zhang Y, Jiang S, Yin QF, et al. Coordinated circRNA biogenesis and function with NF90/NF110 in viral infection. Mol Cell (2017) 67:214-27 e217. doi: 10.1016/j.molcel.2017.05.023

143. Xu S, Xiao S, Qiu C, Wang Z. Transcriptome-wide identification and functional investigation of circular RNA in the teleost large 
yellow croaker (Larimichthys crocea). Mar Genomics (2017) 32:71-8. doi: 10.1016/j.margen.2016.12.004

144. He L, Zhang A, Xiong L, Li Y, Huang R, Liao L, et al. Deep circular RNA sequencing provides insights into the mechanism underlying grass carp reovirus infection. Int J Mol Sci. (2017) 18:1977. doi: 10.3390/ijms180 91977

145. Shen Y, Guo X, Wang W. Identification and characterization of circular RNAs in zebrafish. FEBS Lett. (2017) 591:213-20. doi: $10.1002 / 1873-3468.12500$

146. Cadena C, Hur S. Antiviral immunity and circular RNA: no end in sight. Mol Cell (2017) 67:163-4. doi: 10.1016/j.molcel.2017.07.005
Conflict of Interest Statement: The authors declare that the research was conducted in the absence of any commercial or financial relationships that could be construed as a potential conflict of interest.

Copyright $(0) 2018$ Wang, Jiang, $W u, Y u$, Chang, Li and Wang. This is an open-access article distributed under the terms of the Creative Commons Attribution License (CC $B Y)$. The use, distribution or reproduction in other forums is permitted, provided the original author(s) and the copyright owner(s) are credited and that the original publication in this journal is cited, in accordance with accepted academic practice. No use, distribution or reproduction is permitted which does not comply with these terms. 